Volume 8, No. 7, July - August 2017

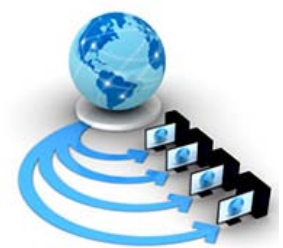

International Journal of Advanced Research in Computer Science

RESEARCH PAPER

\title{
COMPUTATIONAL METHOD OF GALAXY FORMATION IN STANDARD MODEL OF COSMOLOGY
}

\author{
Jaswant K. Yadav \\ Department of Physics, \\ Central University of Haryana \\ Mahendergarh, Haryana, India
}

\begin{abstract}
We present a computational model to study the formation of galaxies in the Universe. The model is implemented in the form of semi-analytic prescriptions describing various physical processes on top of dark-matter-only N-body simulations. Merger trees of dark matter haloes are obtained from the simulations and baryonic physics is then implemented in each halo. This involves models of star formation and evolution, gas heating and cooling, supernova and AGN feedback, dynamical friction effects on satellite galaxies, and major and minor mergers of galaxies. The model is calibrated with observations. We establish the validity of the model via its comparison with the Luminosity function and Stellar Mass function of galaxy population observed with the help of large telescopes.
\end{abstract}

Keywords: galaxies:evolution - galaxies:high redshift - large-scale structure of Universe - method : computational

\section{INTRODUCTION}

The process of galaxy formation occurs when the primordial gas in overdense regions of the matter distribution cools and collapses to form stars (Hoyle 1953 [1]; Rees \& Ostriker 1977 [2]; Silk 1977 [3]; Binney 1977 [4]). The formation of these first stars leads to emission of highly energetic radiation that helps in ionizing the space between different galaxies known as inter-galactic medium (IGM). The time period of initially neutral IGM converting itself into an ionized medium is referred to as the 'epoch of reionization' (see e.g. Loeb \& Barkana 2001 [5]). Prior to this period, almost all hydrogen in the Universe is in the neutral atomic form (H I ). Through this period, hydrogen is ionized so that we are left with almost no H I in the IGM and all that is left of the $\mathrm{H}$ I is its component that remains in the interstellar medium (ISM) of galaxies whose density is very much different from the density of IGM. In the ISM, this remaining Hydrogen acts as the raw material for star formation. In essence this component of neutral gas plays an important role in the future age of galaxies. It is therefore imperative to know how much neutral hydrogen there is in the universe at these redshifts as well as how this is distributed on large scales.

Modelling the distribution of $\mathrm{H} \mathrm{I}$ at high redshifts is a difficult task. Different approaches have been followed to solve this problem. These approaches fall into three basic categories: (1) Empirical modelling of galaxy formation, which relies upon observations of $\mathrm{H}$ I in sky, (2) Hydrodynamical simulations of $\mathrm{H} \mathrm{I}$, in which gas dynamical cosmological simulations are used to model $\mathrm{H}$ I content of galaxies from first principles, and (3) semi-analytic modelling, in which physically motivated analytic prescription are used with results of numerical simulations of large scale structure to understand the nature and distribution of $\mathrm{H} \mathrm{I}$ in the Universe.

In this paper, we propose a semi-analytic model of galaxy formation which can also be used to study the distribution of $\mathrm{H} \mathrm{I}$ in the universe. This type of model is currently one of the most favoured route to making predictions for H I (Baugh 2006 [6]; Power, Baugh \& Lacey 2010 [7]; Kim et al. 2011 [8]). Different astrophysical processes involved in formation of galaxies are taken care of with the help of simple but physically motivated prescriptions. At any given time, the amount of cold gas in a galaxy is determined by the rates of various processes that decrease and increase it. The implementation of these processes is done on halo-by-halo obtained as a results of gravity-only cosmological N-body simulations. The greatest advantage of this approach is its computational speed. These calculations can cover millions of haloes in a large cosmological volumes in a short time, thereby allowing a detailed study of the parameter space. Baugh et al. (2004) [9] first presented predictions for the mass function of cold gas in galaxies using one particular semi-analytic model. They later compared their results with three other models, which differed from their original models in the details of various physical prescriptions (Power, Baugh \& Lacey 2010 [7]). Despite the differing prescriptions, the predictions of all these models show generic features (Benson 2011 [21])

\section{SEMI-ANALYTIC MODEL}

The development of this model started in the seminal works by White \& Frenk (1991) [10], Kauffmann, White \& Guiderdoni (1993) [11], Kauffmann et al. (1999) [12], Springel et al. (2001) [13], De Lucia, Kauffmann \& White (2004) [14], and Croton et al. (2006) [15]. The reader is referred to those papers for more general information on semi-analytic techniques. There are two main parts in the semi-analytic modelling of galaxy formation: (A) constructing dark matter merger trees from a sequence of outputs from dark-matter-only cosmological N-body simulations, and (B) the implementation of actual recipes for the physics of galaxy formation on top of these merger trees. We now describe both of these in order

IIA : N-Body Simulations : We have used Millennium II simulations (Springel 2005 [16]) merger trees. It is an arrangement of about 10 billion particles in a 100 
Megaparsec box simulation. The particle outputs are arranged at 68 snapshots between redshift 127 and 0 . There are in total about 10 Million merger trees describing the evolution of each subhalo in Millennium II simulation.

\section{IIB : Galaxy Formation :}

We have implemented the semi-analytic model of galaxy formation, by supplementing the catalogues of dark matter haloes with the notion of a galaxy population with physical properties given by semi-analytic prescriptions. Such an approach has also been discussed in detail in Kulkarni G. (2011)[20] and the reader is referred to the that text for further references. As is usual in such an approach, we assume that each dark matter halo contains exactly one 'central galaxy. The position of central galaxy inside the halo is given by the central particle of the halo. Other than the central galaxy, a halo can also be occupied by one or more 'satellite galaxies,' where the positions of each one of them is given by one of the particles of the halo. Satellite galaxies are galaxies that had been central galaxies themselves in the past, but their haloes have merged at some previous time with the larger halo that they now reside into. Satellite galaxies orbit in the halo and are assumed to merge with the central galaxy on a time scale known as dynamical friction time-scale.

Hence we deal with a population of central galaxies and satellite galaxies, each attached to the position of a simulation particle at every output time in our simulation. Starting at the first output snapshot which is at high redshift (of around 10 in our case), when the first haloes have formed, we initialize the galaxy population with a set of central galaxies, one for each halo, with mass of stars and cold gas, and luminosity being equal to zero. The physical properties of these galaxies are then evolved to the next output time, where we obtain a new galaxy population based on a combination of semi-analytic prescriptions and the merging history of dark matter haloes. Propagating this scheme forward in time, from output to output, we obtain the galaxy population at the present time, and at all output times at higher redshift.

We now describe the prescriptions used in this evolution. We begin by improving the definition of the progenitordescendant relationship used in deriving the merger trees. According to the improved criterion, a halo $\mathrm{H}_{\mathrm{P}}$ at redshift $\mathrm{z}_{\mathrm{P}}$ is defined to be a progenitor of a halo $H_{D}$ at a lower redshift $\mathrm{z}_{\mathrm{D}}<\mathrm{z}_{\mathrm{P}}$ only if (1) at least half of the particles of $\mathrm{H}_{\mathrm{P}}$ are contained in $\mathrm{H}_{\mathrm{D}}$, and (2) the central particle of $\mathrm{H}_{P}$ is contained in $\mathrm{H}_{\mathrm{D}}$. At low redshifts, this change typically reduces the number of haloes being considered to host a galaxy in merger trees by about $20 \%$.

Next, beginning with the galaxy population at redshift $z_{P}$ , we generate the galaxies of the new population at redshift $\mathrm{z}_{\mathrm{D}}$ based on the merging history of the haloes. Using the halo catalogue at redshift $z_{P}$, we create an 'initial' population of galaxies at redshift $\mathrm{z}_{\mathrm{D}}$ as follows :

- $\quad$ Each galaxy at $\mathrm{z}_{\mathrm{P}}$ is assigned to its new halo at $\mathrm{z}_{\mathrm{D}}$.

- Each halo at $z_{D}$ selects as its central galaxy, the central galaxy of its most massive progenitor. Central galaxies of other progenitors become satellite galaxies of the new halo.

- If a halo has no progenitors, a new central galaxy is created at the position of its most-bound particle.

Once this initial population of galaxies is created at redshift $z_{D}$, evolution of properties of these galaxies for the time between the two snapshots is governed according to our physical prescriptions to obtain the 'final population' of galaxies at redshift $\mathrm{z}_{\mathrm{D}}$.

We model the following physical processes: (1) cooling of hot gas by radiative processes on to central galaxies, (2) transformation of cold gas into stars by the our adopted method of star formation, (3) reheating of cold gas leading to the ejection of gas out of the halo, by supernova feedback, (4) Loss of orbital energy of satellite galaxies and their further merging with central galaxies, (5) evolution of photometric properties galaxies, and (6) Active galactic nuclei. The main features of these prescriptions are as follows.

\section{Gas Cooling}

Cooling of hot gas is modelled according to standard ideas presented in White \& Frenk (1991) [10]. According to this model, each dark matter halo collapses with an amount of baryons equal to the cosmic baryon-to-dark-matter ratio. The diffuse gas with primordial composition contains the baryons, to which metals are added only upon successful completion of process of star formation inside these haloes. Upon entering the dark matter halo, the gas is assumed to shock heat itself to the virial temperature of the halo. Cooling of hot gas in haloes depends on the redshift and on the depth of the halo's potential well that is a measure of temperature of the halo. In low mass haloes at high redshift, the shocked gas cools rapidly and settles onto a central object. On the other hand, in high mass haloes at low redshift, gas spreads in the form of a quasi-static hot atmosphere that extends to the halo virial radius. We computationally model all these processes.

\section{Star Formation}

We use the prescription presented by De Lucia, Kauffmann \& White (2004) [14] as our star formation recipe. The gas which had become cold due to the processes explained in the last subsection now becomes available as a fuel to the process of star formation. The process of star formation also heats up the cold gas as explained in next subsection.

\section{Reheating and feedback}

Supernova explosions play an important role in the evolution of the Interstellar Medium. The amount of cold gas reheated by supernova explosions can be estimated using energy conservation arguments which assumes that the total amount of gas can not exceed the mass of initial haloes multiplied by baryon fraction of the Universe. Our prescription for supernova feedback is as used by Croton et al. (2006)[15].

\section{Mergers}

Mergers of galaxies are common in the hierarchical picture of galaxy formation. In our model, we do not calculate the morphological evolution of galaxies. We treat merger of a satellite galaxy with a central galaxy by using the dynamical friction time-scale suggested by the N-body studies of Navarro, Frenk \& White (1995) [17]. When a small satellite galaxy merges with a large central galaxy, its stars, cold gas and metals are simply added to the central galaxy. If the ratio of baryonic masses is greater than 0.3 , we implement a different approach in which a fraction of cold gas in the merger remnant is instantaneously consumed as starburst in the halo. 


\section{Spectrophotometric evolution}

We use stellar population synthesis models to calculate photometric properties of galaxies in our model. In these models, a stellar population is chosen according to an initial mass function and then each star is evolved along theoretical stellar evolutionary tracks. We use the STARBURST99 code (Leitherer et al. 1999 [18]; Vázquez \& Leitherer 2005 [19]) with suitable parameters.

\section{AGN feedback}

Each galaxy contains a supermassive black holes in our model. The associated AGN feedback in our work is based on the work of (Croton et al. 2006 [15]). We assume that the quasar mode feedback is adequately treated in our model for starbursts during galaxy mergers.

\section{Calibration}

We calibrate free parameters in our model to reproduce observed properties of our host galaxy. This is because most of the observed properties of galaxy are obtained for our own galaxy only. A similar approach has also been followed in previous semi-analytic models (see e.g. Benson 2011 [20]). We also check that we get the right normalization and slope for mass and intrinsic luminosity relation. The free parameters in our model and their values are as follows:

- Parameters determining star formation efficiency: We set these to 0.09 and 2.2 respectively. The first parameter influences the gas fraction of galaxies and the second controls the dependence of the gas fraction on galaxy mass.

- The supernova feedback reheating efficiency and the ejection efficiency are set to 3.5 and 0.35 respectively. These two parameters control the slope of the mass, intrinsic-luminosity relation used as a calibration. Increased feedback makes the slope of this relation steeper by reducing the luminosity of low mass galaxies.

- The retention parameter $\mathrm{R}$ is also available from population synthesis models. It has a value of 0.3 in our model. This parameter has a very negligible influence on the luminosity function of galaxies.

Having specified our semi-analytic model and its implementation, we now proceed to study its results in the next section.

\section{RESULTS AND COMPARISON WITH OBSERVATIONS}

\section{A. Luminosity function of galaxies}

Our model can provide us the luminosity emitted by each galaxy in the simulation. We can count the galaxy between a range of absolute magnitude and then bin them to produce luminosity function of galaxies. Such a task can as well be performed with the modern observation. This gives us a tool to check whether our model is able to reproduce the observational results.

Figure 1 represents the comparison between luminosity function obtained from our model and observations.

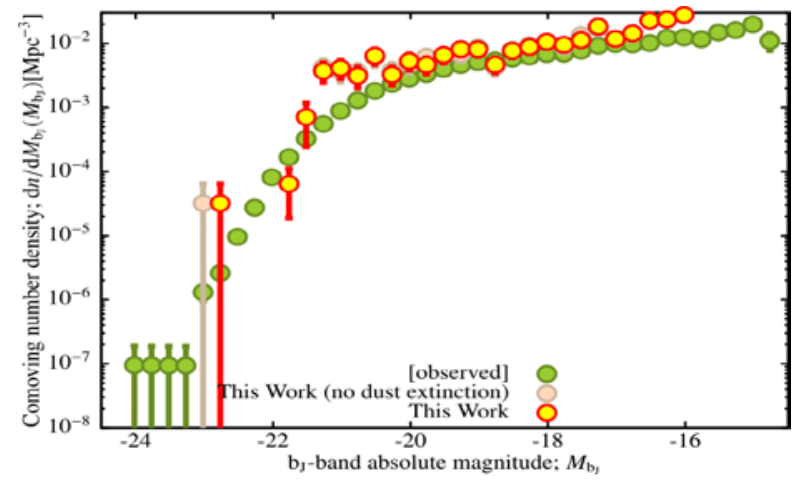

Figure 1. The B-band luminosity function in our simulations is shown by the yellow filled circles with error bars. The green filled circles represent the best fit Schechter function to the B-band luminosity function from the $2 \mathrm{dF}$ Galaxy Redshift Survey (Norberg et al. 2002). We also plot the points where dust extinction has not been included in the model. The $\mathrm{x}$-axis represents the decreasing brightness of galaxies from left to right.

\section{B. Stellar Mass Function}

One more tool to test the outcome of our model is to plot the mass of stars in galaxies as obtained from observations as well as our model. Here we choose galaxies within a particular mass limit in our model and count the number density of galaxies having stellar mass within a particular range. The comparison of our work with observations is shown in Figure 2.

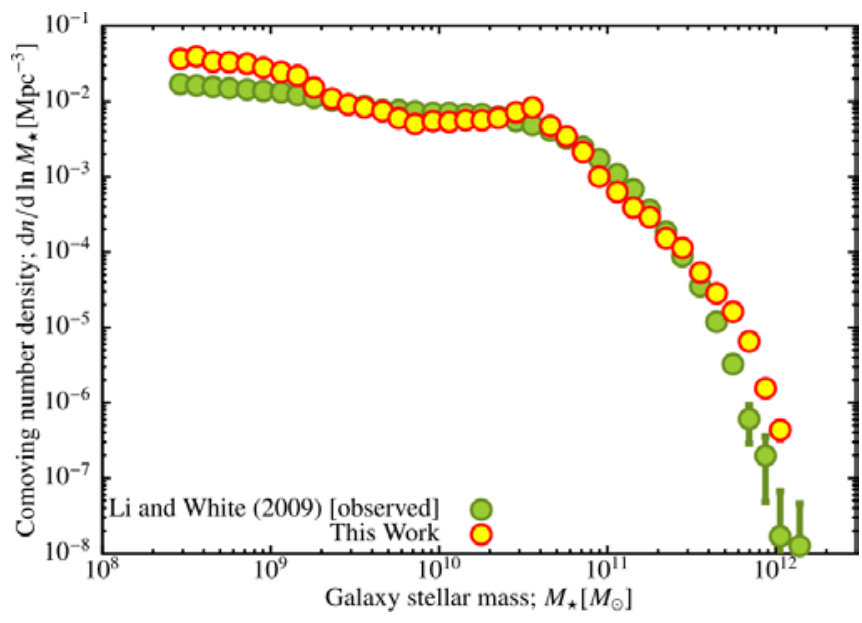

Figure 2. Stellar Mass function of our model (yellow circles) compared to its counterpart from astronomical observations(green dots)

\section{CONCLUSIONS AND FUTURE OUTCOMES}

We have presented a semi analytic model of galaxy formation where the necessary prescription for various physical processes occurring in the process of galaxy formation have been included. This is computationally intensive work, the output of which compares well with the observations as indicated by Figure 1 and Figure 2 above. The code to perform these tasks have been written in combination of fortran as well as $\mathrm{C}$ language and have been run on multi core CPU as well as GPU cluster machines. We 
believe that outcome of this model can be well applicable to the study of neutral hydrogen in the Universe which will also be probed by experimental groups like Square Kilometre Array (SKA skatelescope.org) and Giant meter radio wave telescope(GMRT : gmrt.ncra.tifr.res.in).

\section{ACKNOWLEDGEMENT}

I would like to thank Girish Kulkarni and J.S. bagla for many very useful discussions that I had during the course of writing this code and subsequent analysis.

\section{REFERENCES}

[1] Hoyle F., 1953, "On the Fragmentation of Gas Clouds Into Galaxies and Stars”, Astrophysical Journal, 118, 513

[2] Rees M. J., Ostriker J. P., 1977, "Cooling, dynamics and fragmentation of massive gas clouds - Clues to the masses and radii of galaxies and clusters", Monthly Notices of Royal Astronomical Society, 179, 541

[3] Silk J., 1977, “On the fragmentation of cosmic gas clouds. I - The formation of galaxies and the first generation of stars”, Astrophysical Journal, 211, 638

[4] Binney J., 1977, "The physics of dissipational galaxy formation”, Astrophysical Journal, 215, 483

[5] Loeb A., Barkana R., 2001, "The Reionization of the Universe by the First Stars and Quasars”, Annual Reviews of Astronomy \& Astrophysics, 39, 19

[6] Baugh C. M., 2006, "A primer on hierarchical galaxy formation: the semi-analytical approach”, Reports on Progress in Physics, 69, 3101

[7] Power C., Baugh C. M., Lacey C. G., 2010, "The redshift evolution of the mass function of cold gas in hierarchical galaxy formation models”, Monthly Notices of Royal Astronomical Society, 406, 43

[8] Kim H.-S. et al., 2011, "The spatial distribution of cold gas in hierarchical galaxy formation models", Monthly Notices of Royal Astronomical Society, 414, 2367

[9] Baugh C. M. et al., 2004,"Predictions for the SKA from hierarchical galaxy formation models" in Science with the Square Kilometer Array, edited by C. Carilli and S. Rawlings, Elsevier, Amsterdam”, New Astron. Reviews, 48, 1239

[10] White S. D. M., Frenk C. S., 1991, Astrophysical Journal, 379,52

[11] Kauffmann G., White S. D. M., Guiderdoni B., 1993, Monthly Notices of Royal Astronomical Society, 264, 201

[12] Kauffmann G. et al. 1999, Monthly Notices of Royal Astronomical Society, 303, 188

[13] Springel V. et al., 2001, Monthly Notices of Royal Astronomical Society, 328, 726

[14] De Lucia G., Kauffmann G., White S. D. M., 2004, Monthly Notices of Royal Astronomical Society, 349, 1101

[15] Croton D. J. et al., 2006, Monthly Notices of Royal Astronomical Society, 365, 11

[16] Springel, V., White, S. D. M., Jenkins, A., et al.\ 2005, Nature, 435, 629

[17] Navarro J. F., Frenk C. S., White S. D. M., 1995, Monthly Notices of Royal Astronomical Society, 275, 720

[18] Leitherer C. et al., 1999, Astrophysical Journal Supplements, 123, 3

[19] Vázquez G. A., Leitherer C., 2005, Astrophysical Journal, 621, 695

[20] Kulkarni G. 2011, Ph.D. Theses, http://hdl.handle.net/10603/4712

[21] Benson, A. J. 2012, New Astronomy, 17, 175 